\title{
User Status Sharing for Better Communication, Time Management and User Experience Using Status Wheel Smartphone Application
}

\author{
AbdulrahmanAlkandari $^{\mathrm{a}}$, Adel Alfouderi ${ }^{\mathrm{b}}$, AbeerAlameeri $^{\mathrm{c}}$, Dana Al-Ademd ${ }^{\mathrm{d}}$, \\ Mustafa Ali Abuzaraida ${ }^{\mathrm{e}}$ \\ ${ }^{a, b, c}$ Computer Department-College of Basic Education-PAAET Kuwait \\ ${ }^{\mathrm{d} C}$ Computer department, CPE, PAAET, Kuwait \\ ${ }^{\mathrm{e}}$ School of Computing, College of Arts and Sciences, Universiti Utara Malaysia, Sintok, Kedah, Malaysia, \\ aaam.alkandari@paaet.edu.kw, ${ }^{\mathrm{b}}$ ak.alfoudery@paaet.edu.kw, ${ }^{\mathrm{c}}$ ae.alameeri@ paaet.edu.kw, ${ }^{\mathrm{d}}$ aladem.dana@gmail.com, \\ eabuzaraida@uum.edu.my
}

Article History: Received: 10 November 2020; Revised 12 January 2021 Accepted: 27 January 2021; Published online: 5 April 2021

\begin{abstract}
: this paper presents a new social-networking concept and solution for effective appointment-scheduling and status communication between groups of people. The concept is driven by the widespread adoption of social-networking platforms among people as a result of daily internet usage. People became connected most of the time with other users of a social network. According to studies and statistics, usage of smartphone applications for social networking is increasing rapidly in the past years. This paper introduces Status Wheel application as a solution to some of the common problems facing social network users via smartphones. Such problems include the issue of a well-managed time schedule and appointments. This may lead to more people going out at peak hours to meet a teacher or a co-worker, which creates avoidable traffic-jams. So, an effective platform for quick user status updates and communication with other concerned individual is needed. This paper introduces Status Wheel smartphone application. It promotes communication through sharing of users' current status. Convenient user-experience is studied and given paramount importance as well.
\end{abstract}

Keywords: Social Network, Time Management, Status, Mobile Application

\section{Introduction}

The rapid growth of technology is affecting people's life vastly nowadays. Usage of the Internet is moving towards more employment of social media and mobile applications. The use of mobile applications affects time management positively.

Internet has become a daily necessity. Statistics show that the numbers of internet users reached to $23.2 \%$ of global population in 2008 and increased up to $38 \%$ with a number of 2.7 billion users in 2013. Internet users grew annually to reach 3 billion users by the year of 2015[1]. Internet users are constantly being driven to social media. According to a global study, it shows that no less than $60 \%$ of active Internet users spend their time on social networks [2].

Social media use the mobile technologies and web based applications to make communications more reactive. It encourages online information sharing among wide area of people. Furthermore, it increases the communication skills of users. A study of 138 social network users shows that $68.5 \%$ of these users think of social network as a major communication tool. Additionally, majority of users said that they prefer social networking to other social media tools, like Wikis, video sharing, blogs... and the reason of this preference is the usefulness of social media. It is an interactive medium, source of information and bridges the communication gap. Social media benefits both business and educational sectors in time managements and communications [3]. Online workers find E-mail and the Internet to be the most useful communication and information tool [4].

Because of the increasing number of people using social networking, people moved towards holding mobile devices and smartphones more. Statistics show that $85 \%$ of social networks users said that mobile devices are a central part of everyday life. The average smartphone user spends about 3.3 hours a day on their smartphones [5].

Furthermore, Smartphones have many benefits. Multi functionality, ease of use, mobility and connectivity are just some to mention. Users can also make use of many applications without extra cost. 
Mobile applications usage grows continuously as the need to stay connected to the Internet becomes part of the daily lives of people. In the past few years the overall unique mobile applications audience increased by $85 \%$ from 2011 to 2012 then to $37 \%$ by 2013 as shown in figure 1. [6, 7].

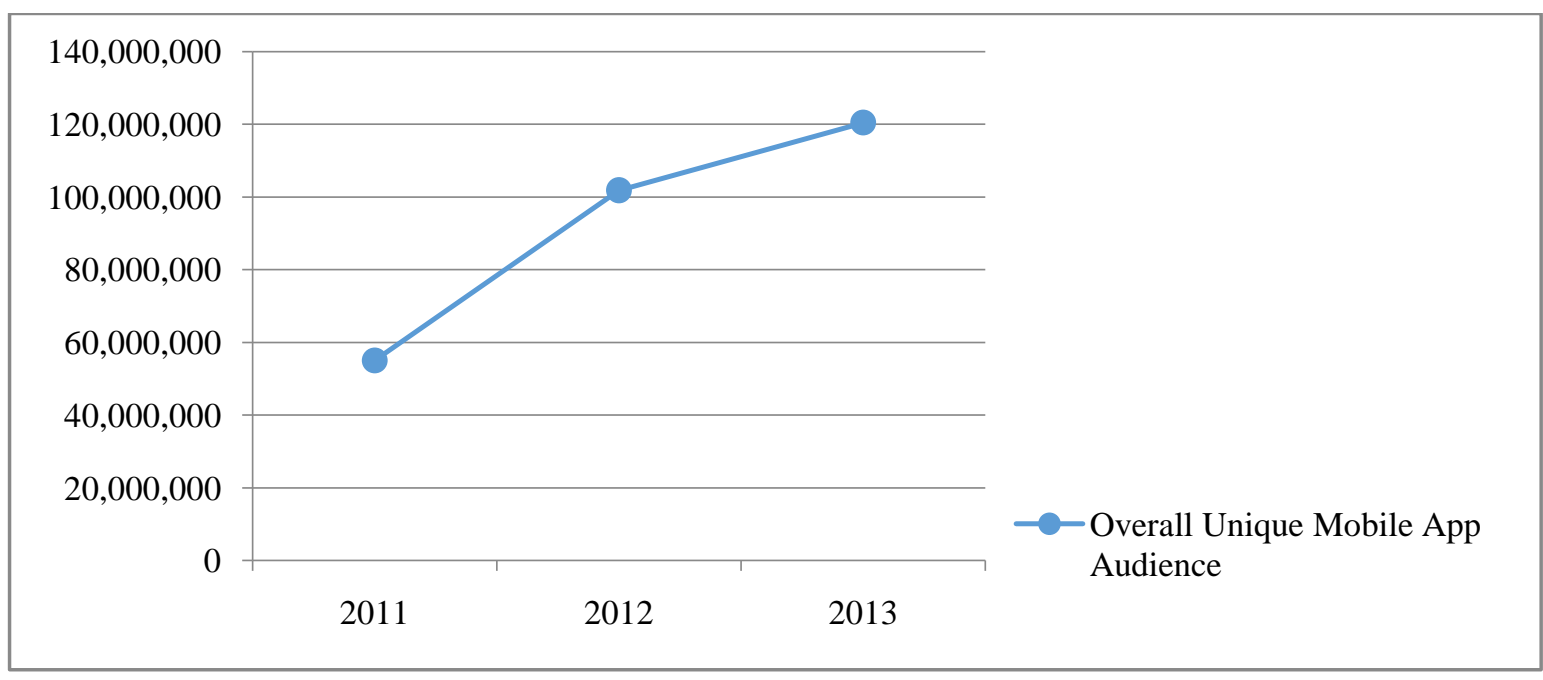

Figure 1: Overall Unique Mobile App Audience [6][7].

Mobile applications are actual upgradable programs that can be installed directly on the device without using browsers. Social messaging applications provide the user with the ability of sending and receiving messages conveniently. Response time, which is the time that takes the program to response for a specific command, should be within few milliseconds in reaction time. Ease of access allows the user to be easily accessible to the application's expenses and information. The messaging applications also have some advanced features such as notes and media files sharing [8].

Privacy is a major concern when using social media applications. Most people may not like to share personal information publicly. Misuse of social applications may interfere on one's privacy. This leads to use fake personal information which misleads people and does not comply with the intended purpose of social applications [3]. Popular messaging applications like Facebook, WhatsApp and Twitter have their own privacy policies that protect user information such as client profile picture and client status. [8] [16].

Adopting social media via mobile applications is a helping factor in time management. People are able to make appointments with a single status update. The word status may seem something that has a small role that can be neglected, but in fact, it plays a big role in each and every one day-to-day life. Sharing status through social media aim is to save time and effort. Although most of the social media applications have the status option, it is better to have an application that is specialized for showing status only. The reason for creating this status application is to have a faster access, and a quicker update. Such an application can be very useful for college students, company workers and busy people. This paper will introduce status wheel smartphone application.

\section{Problem Description}

Time is a very important commodity as long as we make the most of it. No productive person should face a time waste that leads to a slowdown in the process of accomplishment. Wasted time is common in many scenarios. For instance in universities where communication between instructors and students could be conflicted by time factors between the two parties. In one case, when students want to meet with their instructor, but they waste their time waiting since the instructor is not available for being in a meeting or elsewhere. This could also occur between instructors themselves. Another instance is in companies where co-workers need to communicate face to face urgently. These small cases might highly create a much larger problem such as traffic jams which is a result of people going out in similar times without prior notice. Another problem is that using variety of social media applications could lead to slowing in operating when sharing the same status across all of the applications. Not to mention, this repetition brings boredom to the user or even delay in status update.

Another problem of using different applications is the different user interfaces and user experience for each one of them, which confuse the users and require additional time to get used to them. 


\section{Related Work}

There are a set of new smartphone applications created to help user co-ordinate status messages and enhance communication. Social media applications are considered the key used to connect the worldwide communication system together. Some examples are: Frindlee, WhatsApp, Facebook and Connecto [14].

Friendlee is a social media application that is used for communication. It has many great features, but it is well known for its capability of publishing context information. It is necessary to have different kind of awareness cues, like status and location to know other's situation and status [9].

WhatsApp is a very popular application for connecting people world widely. It is a free messenger that is designed for sending direct messages in smartphones and it has the status option, which enhances the effectiveness of communication. According to the User Satisfaction Towards Whatsapp study, 38\% of WhatsApp users use the program to keep in touch with others, to share videos, photos and statuses. [10] [15].

Nowadays, social sites are considered the most visited web pages worldwide. Facebook is a leading social site, with more than 90 million active users in 2009. Facebook has taken a pride of over $85 \%$ of market share at colleges and universities. Currently, Facebook users reached more than 800 million active users around the world. This site has the ability to share a real time instant message anytime around the world with 'one click' between different users. Moreover, it provides different statuses that enhance the relationship between different users.[11].some of the main features are: the ability to make connections, status updates, share media and comment on other's content. On an average day, $15 \%$ of users update their status, while $22 \%$ make comments to posts or statuses, $20 \%$ comments on photos, $26 \%$ give' likes' to others And $10 \%$ send private messages as shown in figure 2.[12]

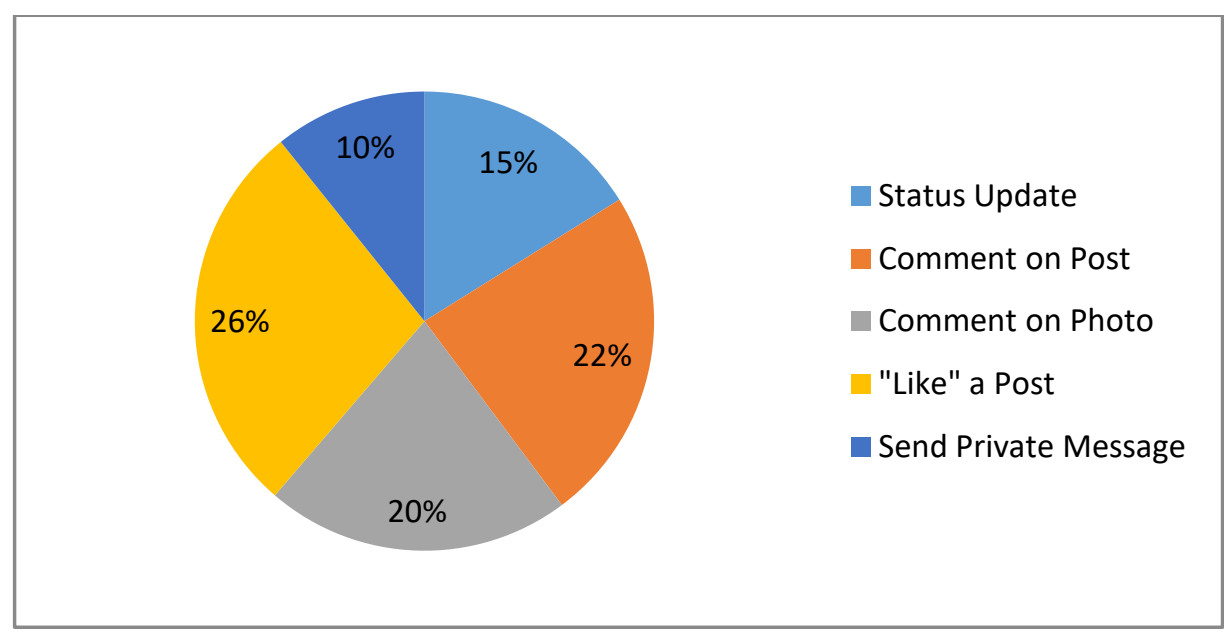

Figure 2: Average Facebook daily usage [12].

Connecto application, status and location sharing application, is an application that allows users to share texts along with individuals' location. It was created to see how people are using the location awareness. Connecto have a main feature which is showing the contacts locations. A user can be texted or called by using the application. The application front-page shows the contacts and their location, status, with the options which allows the user to change from manual to automatic location mode and also messaging features which shows the status time [13].

From the above mentioned studies, it is important to share status with messages and smartphones. It also helps to avoid miscommunication in order of calling someone at the wrong time, late replies, etc.

\section{Application Design}

The fact that the majority of communities are using smartphones and social media applications is an inspiration to create a smartphone application to deliver information much faster. Status Wheel is an interactive application that is specialized in sharing current status with co-workers, groups or friends on the go. Both android and IOS users can download and use this Application freely. It benefits college students and instructors, companies and any busy people. It promotes an exchange of current status between workers, which facilitate communication methods. Status Wheel application also help in time management by saving effort, when knowing the exact current status of the wanted person, user can avoid going to their places without prior planning, which will help in avoiding traffic jams and disorganizing of time. The status wheel application provides a useful and a unique feature that can be very helpful for its users, the ability to change the current status depending on the current location of the user. The user of the application can activate this feature and select on the map their most visited 
locations with a pin. These pins can be linked to specific status; whenever the user reaches these locations the application automatically changes the current status to the set one.

- Status Wheel application helps in organizing time schedules of users. Users can check their followers' status by viewing their profiles in the application instead of going to their places.

Status Wheel application designed to have a circular shape like a wheel. This design brings entertainment while keeping everything professional. The simple design is keen to the eye and makes the learning curve an easy task. The pre-defined status parts make updating one's status faster by just clicks rather than writing status every time. Having the status parts in specific places builds muscle memory over short period of time. Flexibility is an option for the Status Wheel since it is possible to edit parts and change them as desired. Status Wheel application serves specific purpose that makes it simple to use. The application interface designed with clear and specific tools accessible to everyone. All of that made the application user-friendly at most. This part of paper shows the design of the main features of the smartphone application.

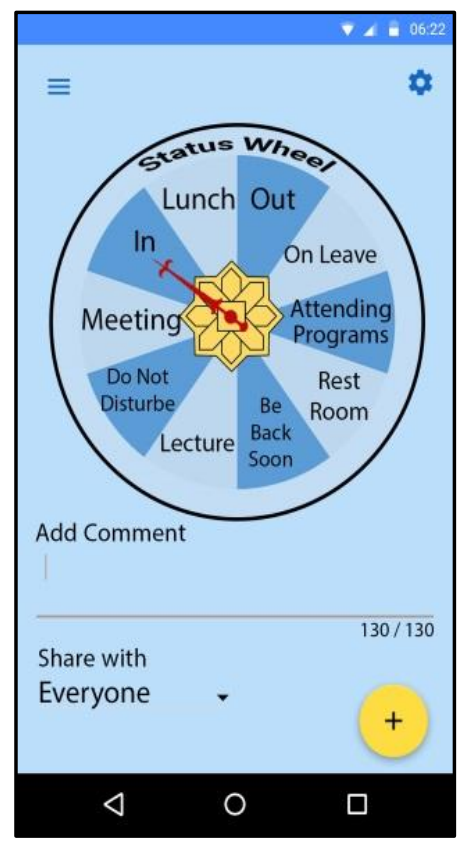

Figure 3: The main interface for sharing status

The first interface appears when launching the application is the user access, login and registration. The user then is redirected to the main page as shown in figure 3. The main page shows the default wheel. Users can choose the status by clicking on the part of the wheel indicating the current status; this presents a faster process of changing the current status. Users can edit the default wheel or delete any unwanted parts by tapping the edit icon link. As an optional feature, user can add comment to the status using the comment box under the Status Wheel. The comments will be displayed along with the status on the user's profile; this service indicates a detailed description of their location. User can change the privacy option in which with whom the status is shared. To share status, user has to press on the share button to post the status.

The main page also has a link to the followers' updates page. This page shows the latest status updates from users the current user is following. It provides easier access to their updates and the ability to comment on their statuses. The side menu list links for the user profile, feed and application settings. 


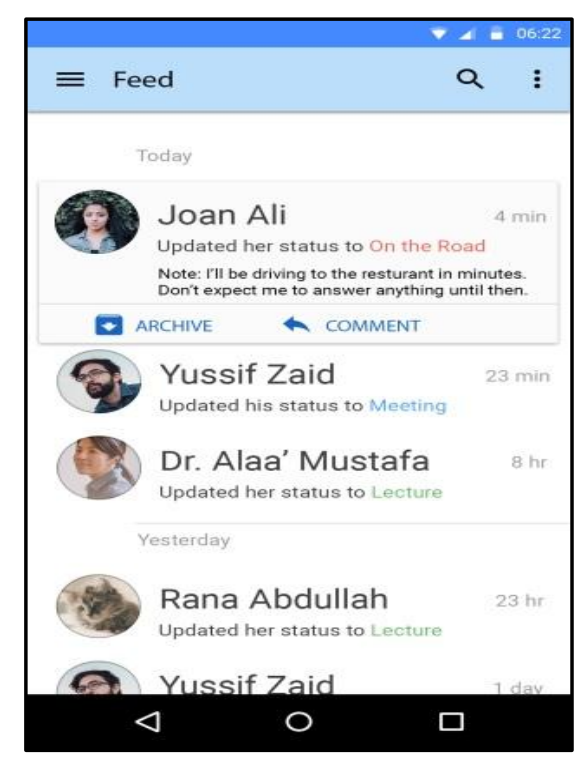

Figure 4: Feed page for followers updates.

The page which the user can see recent status updates by their followings is the feed page as shown in figure 4 . It displays latest status updates whenever another user changes their status. Newer status updates appears on the top. The user status update contains their name, avatar, the current updated status along with any comment they might have added. The user can access their profile for more details. When user sees the status they have an option to archive it in order to hide it from the feed page and notify the user that their status has been seen. Users can also comment on others' statuses.

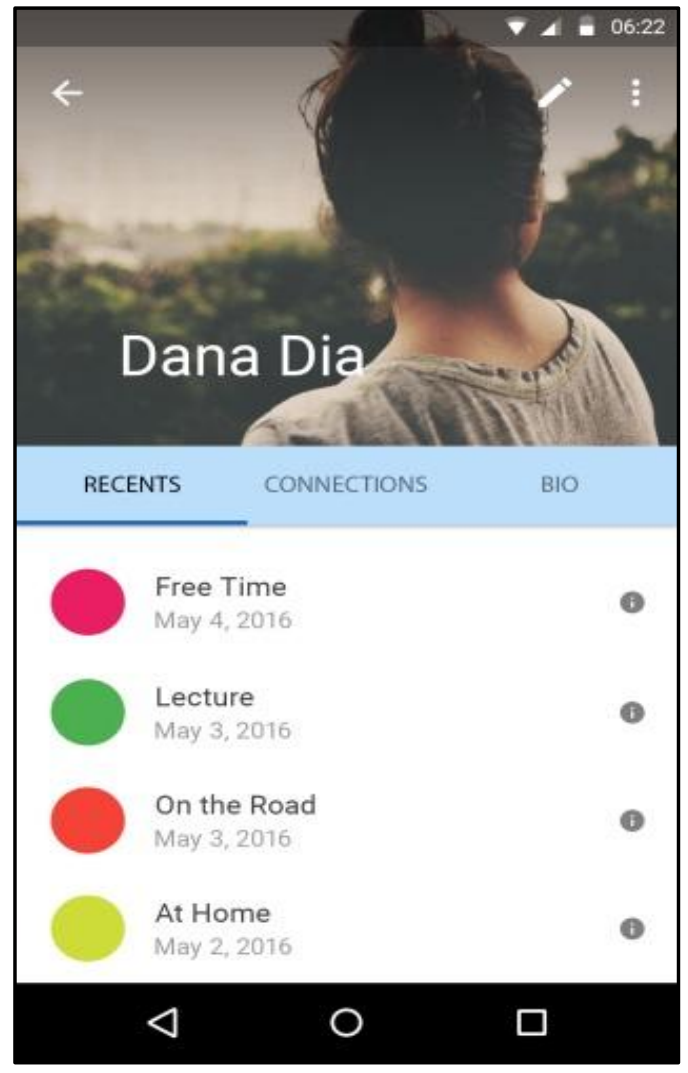

Figure 5: user profile page

The current user profile, as shown in figure 5, includes three sections. The first section displays recent status update of the user. Each status shows any comment the user might have added. It has an icon that expands for further details. The details could be: full date, timestamp, people who archived the status and any comments others have posted to the status. The second section displays the user connections. Lastly is the user bio section. 
User bio contains the first and last name, user E-mail, workplace and any other personal user information the user wishes to share. The interface in general contains user image and their user name.

\section{Implementation}

Status Wheel application is implemented to focus on sharing status as a main process. Other processes the application go through include the following: login and registration process, status feed update process, the Status Wheel editing process, personal profile editing process, search for user process, and following a user process. All these processes work together and complement each other for the benefits of the application. This part of the paper explains the implementation of each process.

The first step when using Status Wheel application is the login process. User is required to input username or E-mail and password to login. If the username or password does not match any correct data, an error will be displayed and the user will have to try again. If the user does not already have an account, they can create a new one via registration. The new user will have to input username, password and E-mail for the new account. User can also reset their password if they lost their existing one. The application is programmed to send a verification E-mail to the user to enable them to reset the password. The user is then redirected to the main page.

The status sharing process starts in the main page. User can share their status with ease by tapping on the selected status from the Status Wheel parts. There is an option to add comment on the new status before sharing.

The first priority for any user is the privacy of their personal information. By improving the application's privacy features, it will increase the trust of a user in the application. Moreover, privacy is about respecting individual's information. Hence, Status Wheel application provides a flexible and more confidential privacy setting such as: specific selections in which the user can benefit from selecting which users to access their personal status. As there are three types of privacy options: everyone, specific users, and my contacts. The user can choose the most flexible option to them for every status share. Status Wheel application has been developed in such a way that it extends the privacy flexibility to avoid unexpected faults. .by default the privacy option for any new status is as defined in the user settings. But it gives the freedom to choose the privacy option per status.

When sharing the new status, it gets stored in the User Status database. After that, the new status will be available to be displayed in different pages such as: profile page and feed page of other users. As described in figure 6 . 


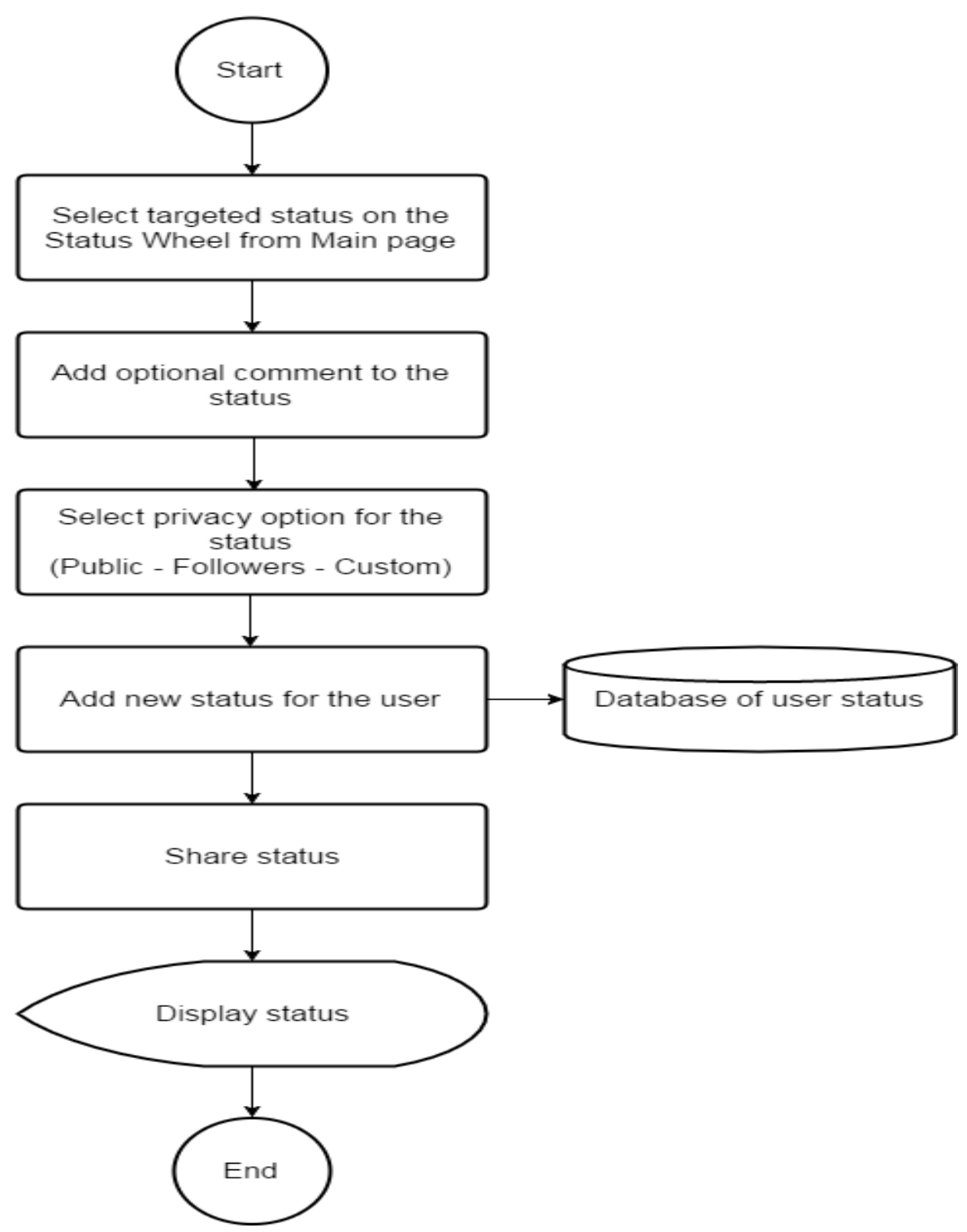

Figure 6: Sharing status process

The edit wheel process is one of the most important processes to the application. It is implemented to give users the freedom to change and edit the status as they wish. It gives the ability to customize the Status Wheel itself. User can start editing the wheel by pressing on the Edit icon in the main page. The edit page has the options to edit a status part or delete it completely. To edit, user can rename the status parts to the desired status. The limit on the status parts is up to ten statuses. User can have less than ten status parts if that all they need by deleting unwanted ones. The recovery option comes associated with the delete option. When any status is deleted, User can recover deleted parts for sure. To do so, a recovery icon appears that allows user to add parts up to ten parts again. Another option in the editing wheel page is the ability to change the colour of each status part of the wheel. When done the updated status wheel will become the default one in use with the new status part values. The process is shown in figure 7.

The other edit process is to edit the user profile. From the current user profile, the edit profile icon will redirect the user to the page where they can edit their profile information. They can add or edit their profile picture, username, company name, full name, E-mail address, age, nationality, location, education, and small bio. Some of these are optional to include but highly recommended for better user experience. The updated information get stored in the user's profile database then displayed to the users. 


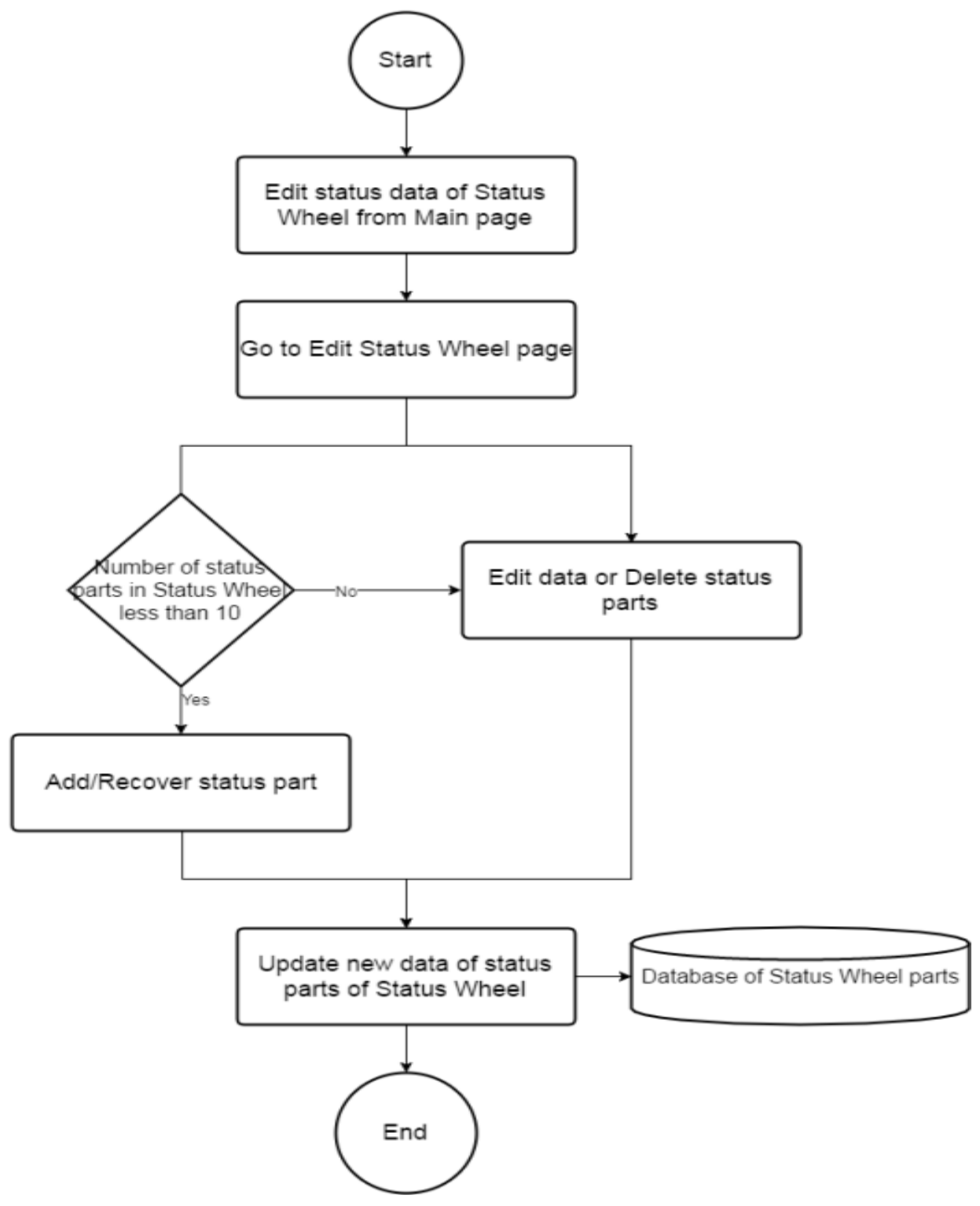

Figure 7: Edit Status Wheel process

When user search for a specific account, the search engine will return relative results of other users accounts. The search algorithm will look for similar terms in the first name, last name, and username fields of profiles then filter the results as requested by user depending on first name, company, last name... Lastly sort the results according to the desired field like the username, company name. The found results will contain the profile image, username... in ascending order and last updated status. Clicking on the desired account will direct the user to that profile. The diagram shown in figure 8 explains the process.

From the search results, user can access the wanted user profile. If the current user is not already following that user, the follow option will be available. The follow process is typical, a follow request is sent to the user, which then they can decide to accept the invitation or deny it. If accepted, a following-follower relationship will be established between them so each can contact the other and receive their updates feed.

The status update feed process is designed to show latest status updates from all of the followings. It retrieves the latest posts from the following users. It is programmed to check for any updated statuses to show it immediately in the feed page. When users see the status they can archive and comment which will appear in the user profile.

Status Wheel application icon is implemented to change whenever the users change their current status. This is implemented by keeping the application running in the background and checking the user status every time the user turns on the mobile screen. The application will check the selected status and display it on the icon in the colour of the status assigned by the user. 


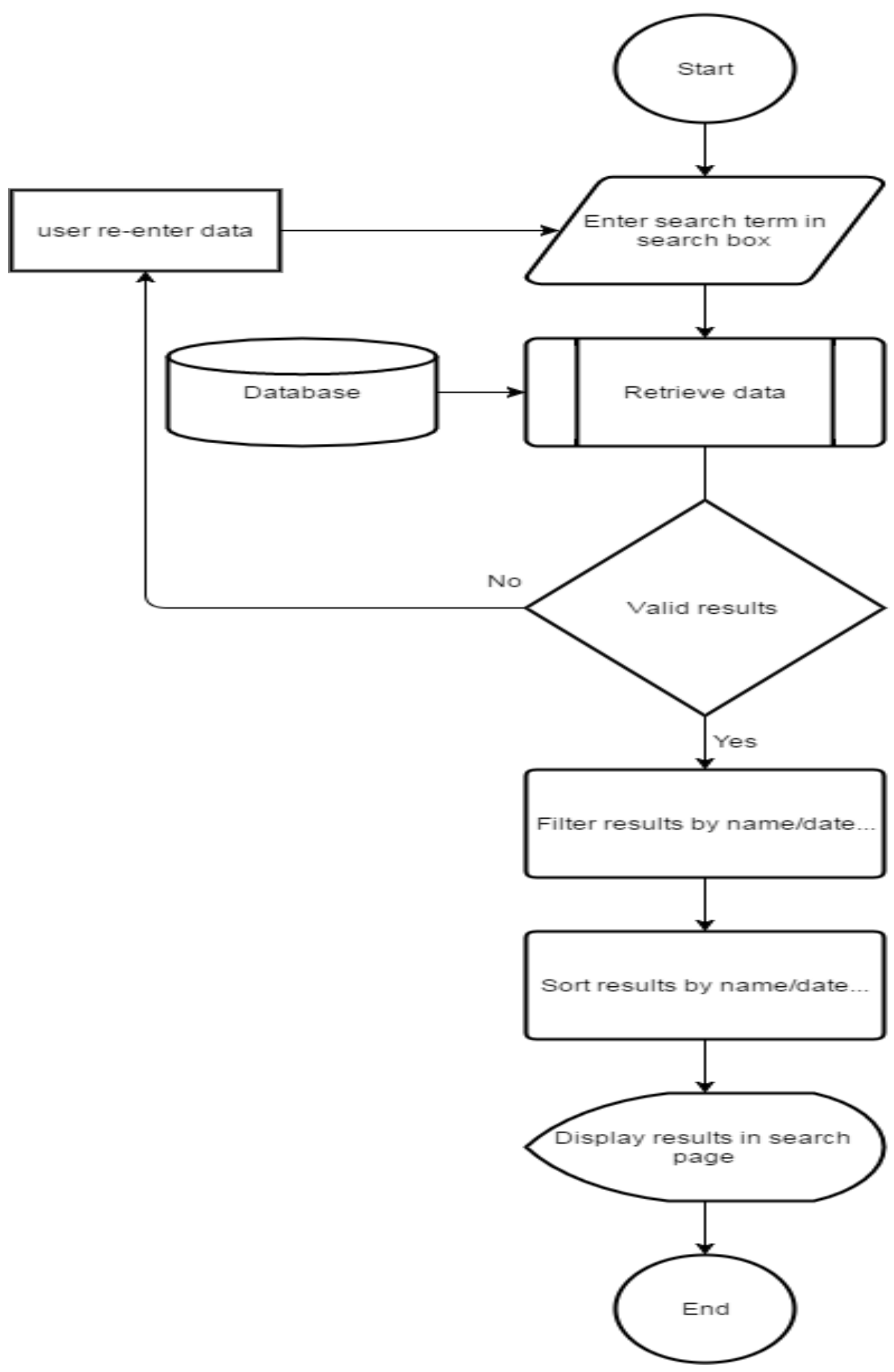

Figure 8: Search process

\section{Results And Discussion}

Status Wheel application gives the ability to search and find any wanted users. Users of this smartphone application can check others statuses, read their comments, comment on their statuses, follow users, archive seen statuses and most importantly update their status.

Status Wheel application benefits users in time management in both sharing statuses and knowing other's statuses. The ability to share current status results in better reach for information that cuts waste of time. This status will be displayed for others to inform them of the user's current status. This knowledge presents a good scheduling factor. For example, when workers or students wants to meet up with their colleagues, they will be able to know their current status and decide accordingly when to go. This will also help to avoid traffic jams in peak hours by avoiding unnecessary drives without prior knowledge of status. All of that result in not wasting time by providing up-to-date information of users' current statuses. The Application provides an additional service 
based on GPS maps, where the user can set specific location to a certain status, whenever the user reaches this location to that location the app automatically share the selected status to that location, all that helps in time management and to solve the problem of frequent users who might forget to update their status.

Status Wheel application is more beneficial than many of other social applications. It focuses on sharing the status while others have sharing status as a side feature. Not to mention that the status sharing process is simpler than writing the whole status every time across different social applications.

Status Wheel application is flexible since it gives the ability for the user to customize the status wheel itself as suits them. Since the user pre-entered their statuses, it results in easier status update and doesn't require them to type it every time.

The application is designed to be simple and on-the-point. The interface is easy to use by most people at any age, education level or technical skills. The series of processes complete each other. This makes reaching the status and users an easy process. All of that resulted in a user-friendly interface.

User profile is reachable from different pages. The search interface, the feed page and the followings in the current user profile are the source pages leading to user profile. In the search page, user can search for username, company name and such. Then the related results displays on the search page. The search result contains avatar, username and the current status. The user profile can also be accessed by clicking on the username from either feed page or followings as shown in Figure 9.

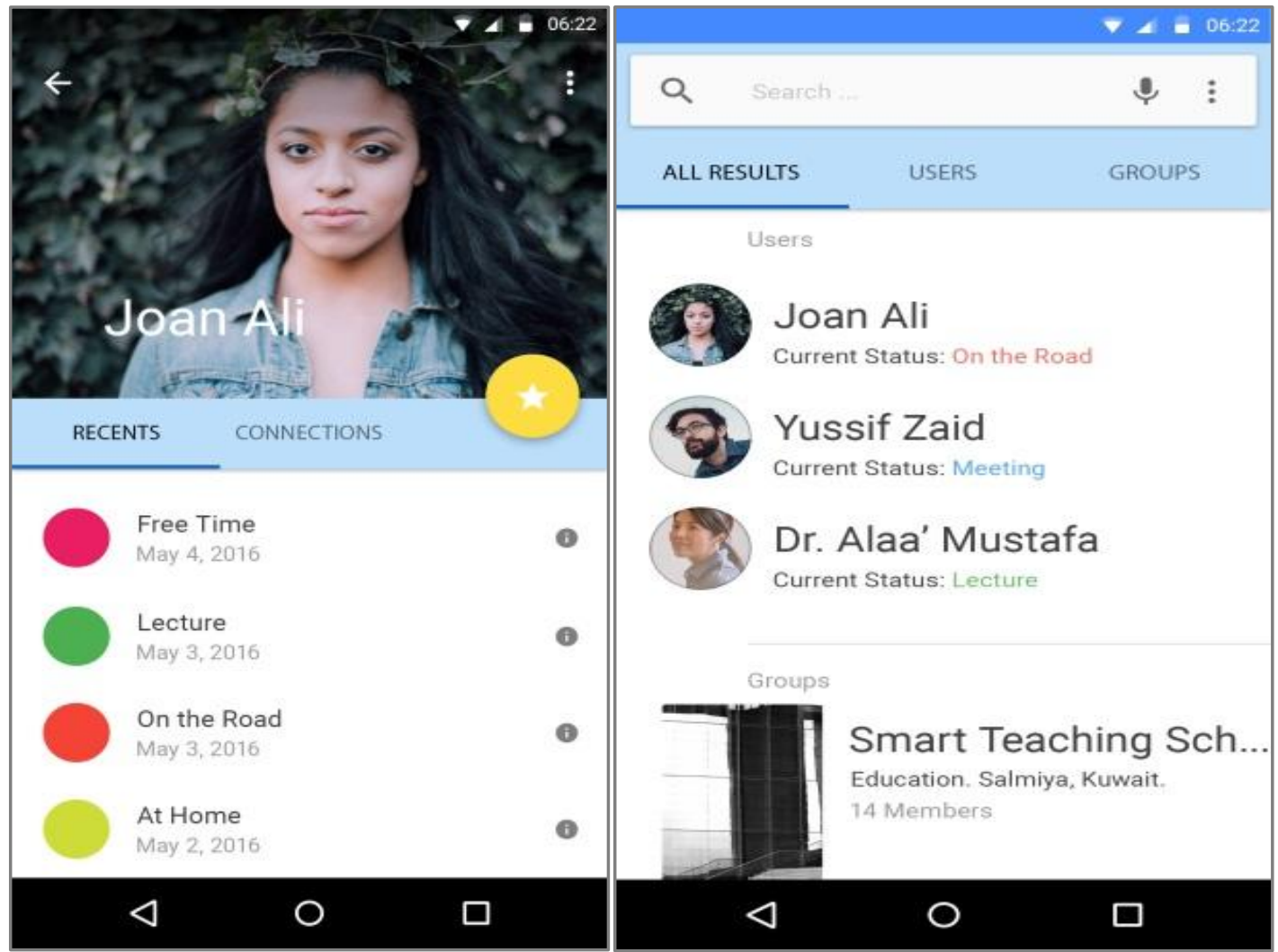

Figure9: user profile as a search result

One of the most important features of the application is the notification badge that appears in the home lock screen. This badge shows the current status on the application icon. Users tend to forget to update their status occasionally so this will act as a reminding tool to the user to keep their status updated. Every time the user changes status it will change accordingly as shown in figure 10. 


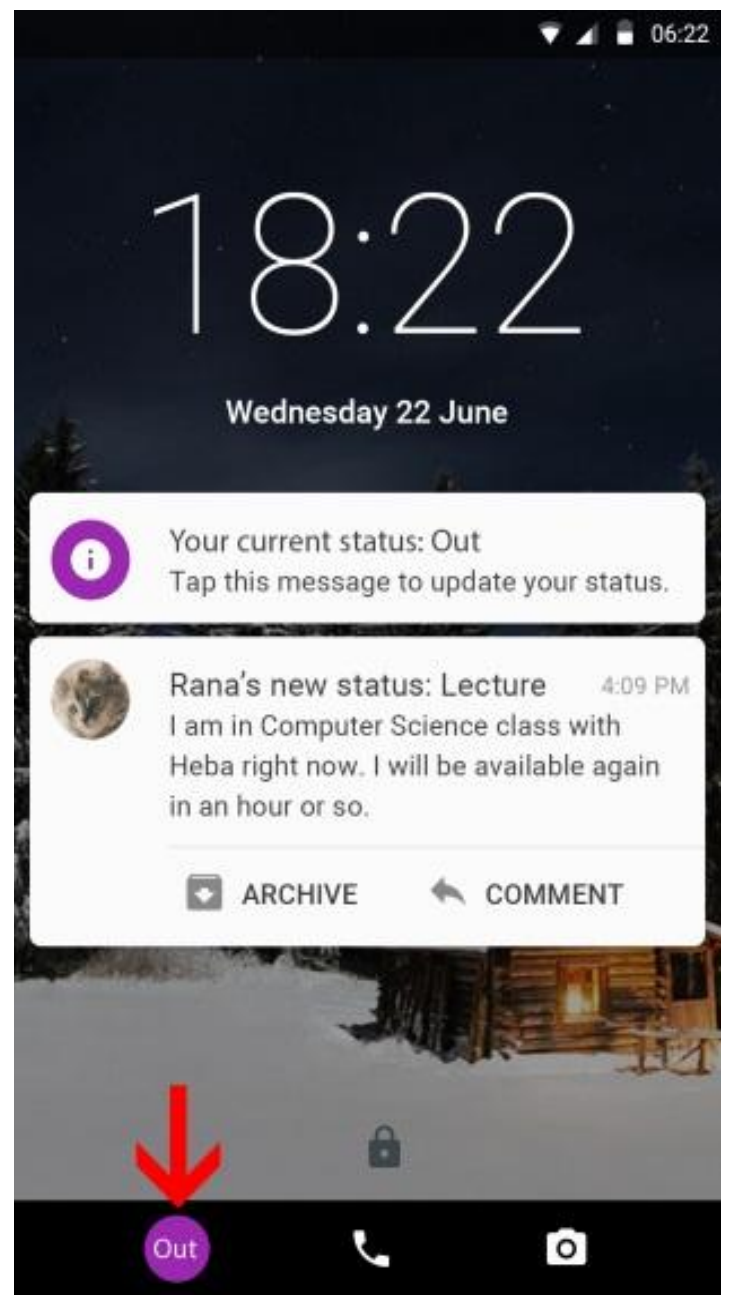

Figure 9: notification in lock screen

The service of linking the map in sharing status helps the users to update their status regularly and speed of the process. this feature can be very useful in scenarios like: how working people have their most visited places to be their work place as a set location; when the users select on the map their work place location , users type a specific status for it like "At work". When the user activates this feature, whenever they reach a location, the application automatically updates the current status depending on the status selected for that location. Same process happens for different locations like home, or even for busy workers, the airport. When the user travels to different city or country, if this feature is activated, the map can specify the location of the users and update their status to the defined city as shown in figure 11 . 


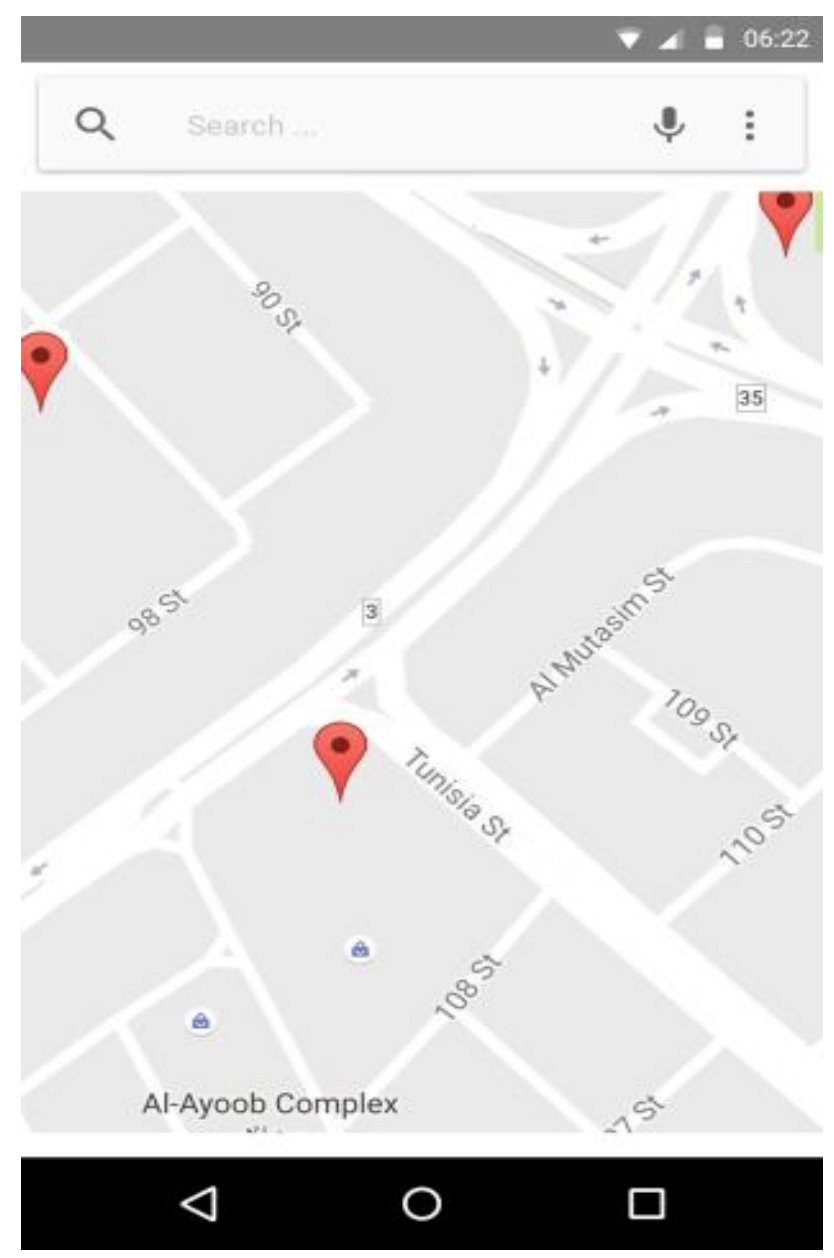

Figure 10: sharing status using maps

\section{Conclusion}

Status Wheel smartphone application is a great tool for organizing. It helps people manage their time better and stay in touch with others. Users in companies or school or even small groups of friends can use Status Wheel application to help keep each other's informed with their statuses. The application can also improve time management by reducing delays and misinformation caused by not knowing the status of someone. The user friendly interface enhances the social experience and cut learning curves of so many other applications. Status Wheel application feature a unique way of updating status using an easy wheel, this wheel help build muscle memory that make sharing a new status a fast and spontaneous task. Being a smartphone application makes it reachable by the majority. The main problem is that some people may not bother to update their status instantly in real time. This is solvable by the notification badge that appears in the lock screen of the mobile. It could be a disadvantage for some that the status is limited in character. This short status title could be vague without further description in comments, which the user might neglect on adding it. This might lead to miscommunication. User behaviour could affect the efficiency when sharing the current status as the post time is open ended.

\section{Future Work}

There are many promising features to be added to Status Wheel application. For starter, the ability to share the current geographical location when updating status can be implemented. Make Status Wheel application connect with other social network applications like Facebook and Twitter to synchronize status sharing across different platforms. A voice recognition system can be added to update status and add a voice comments. The Status Wheel can be customized to increase the numbers of status parts. The feature to add more than one wheel for different uses and the ability to categorize them can be added too. It is also thought to implement algorithms that allows for machine learning so that the application can schedule status changes according to user's status repetition. The application can have an option to set time period for the status with a start and an end time. 


\section{References}

Internet Society.(2015). Internet Society Global Internet Report 2015 Mobile Evolution and Development Of The Internet.

Nielson.(2011).State Of The Media:Social Media Report Q3 2011.

Baruah, T. D. (2012). Effectiveness of Social Media as A Tool Of Communication And Its Potential For Technology Enabled Connections: A Micro Level Study. International Journal of Scientific and Research Publications,2(5), 1-10.

Kristen Purcell, Lee Rainie. Pew Research Center,(2014). “Technology’s Impact On Workers”

Salesforce.(2014). Mobile Behaviour Report Combining Mobile Device Tracking And Consumer Survey Data To Build A Powerful Mobile Strategy.

Nielson.(2012).State of the media:The social media report 2012.

Nielson.(2014).The digital concumer February 2014.

Khatri, S. K., \&Narula, K. (2015).General Maturity Model for Social Messaging Application. In 4th International Conference on Reliability, Infocom Technologies and Optimization (ICRITO) (Trends and Future Directions) (Pp. 1-5).

Ankolekar, A., Szabo, G., Luon, Y., Huberman, B. A., Wilkinson, D., \& Wu, F. (2009).Friendlee: A Mobile Application for Your Social Life. Inproceedings of The 11th International Conference On Human-Computer Interaction with Mobile Devices And Services (P. 27). Acm.

Singh, S., Prasanth, A., \&Subramani, A. K. (2015). User Satisfaction TowardsWhatsapp, Avadi, Chennai. Excel International Journal of Multidisciplinary Management Studies, 5(6), 1-8.

Tham, J., \& Ahmed, N. (2011). The Usage and Implications of Social Networking Sites: A Survey Of College Students. Journal of Interpersonal, Intercultural And Mass Communication, 2(1), 1-11.

Keith N.,Lauren, Lee,Kristen.(2011).Social Networking sites and our lives a project of PewReaserchcenter .

Barkhuus, L., Brown, B., Bell, M., Sherwood, S., Hall, M., \& Chalmers, M. (2008). From Awareness to Repartee: Sharing Location Within Social Groups. In Proceedings of The Sigchi Conference on Human Factors In Computing Systems (Pp. 497-506).Acm.

Sutikno, T., Handayani, L., Stiawan, D., Riyadi, M. A., \&Subroto, I. M. I. (2016).WhatsApp, viber and telegram: Which is the best for instant messaging?.International Journal of Electrical \& Computer Engineering (20888708), 6(3).

Gibson, K. (2020). Bridging the digital divide: Reflections on using WhatsApp instant messenger interviews in youth research. Qualitative Research in Psychology, 1-21.

Kim, M., \& Han, S. (2018). Cognitive social network analysis for supporting the reliable decision-making process. The Journal of Supercomputing, 74(8), 3654-3665 